\title{
Highlights of the ACM Student Research Competition
}

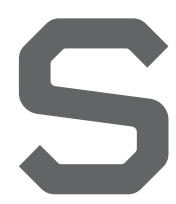

INCE 2003, ACM in conjunction with Microsoft have sponsored research competitions for undergraduate and graduate students in computing. The competitions provide a vehicle for these students to present their original research before a panel of judges and attendees at well-known ACM-sponsored and co-sponsored conferences. The students have the opportunity to experience a research conference, get feedback on their research, meet academic and industrial researchers and other students, and appreciate the practical applications of their research. Student competitors also have the opportunity to sharpen communication, visual, organizational, and presentation skills in preparation for the SRC. Participation by undergraduates may be literally life-changing if they alter their career path to pursue graduate studies and research careers after experiencing a conference and competition.

The following process is used to select the SRC winners:

1. Each student submits an 800word abstract of his or her research. The abstract is evaluated by a minimum of three reviewers. Feedback on the abstract is provided to the students.

2. The students submitting the highest-evaluated abstracts are invited to attend the conference and present their work. Typically, 10 graduate students and 10 undergraduate students are invited to compete at the conference.

3. At the conference, the students present their work in front of a poster. Any conference attendee may come and ask a student about their research. A minimum of five official SRC evaluators assesses each poster.

4. The top five graduate and top five undergraduate students advance to the semifinals. In the semifinals, each student makes a 10-minute pre- sentation of their work followed by a five-minute question-and-answer session to official SRC judges and any conference attendees who attend the open session. At least five judges are assigned to the semifinals.

5. The conference's top three finalists in each category are chosen based on these presentations.

6 . The first-place winners from each conference are invited to compete in the Grand Finals. These students submit an updated 4,000-word paper on their research that is judged by a panel of experts.

The winner of the Grand Finals and their advisors are invited to the Annual ACM Awards Banquet, where they are recognized for their accomplishments and can witness other luminaries in the computing field receive prestigious society awards, such as the ACM A.M. Turing award. The first-, second-, and third-place winners of each conference competition receive cash prizes, medals, ACM student memberships, and recognition among their peers and professorsrecognition that strengthens their résumés. All students who are selected to participate in the conference-level competition receive $\$ 500$ travel funding for the conference.

In 2016-2017, competitions took place at 24 participating conferences sponsored by the following ACM SIGs: SIGACCESS, SIGARCH, SIGCHI, SIGCOMM, SIGCSE, SIGDA, SIGDOC, SIGGRAPH，SIGHPC，SIGMIS， SIGMOBILE, SIGPLAN, SIGACT, SIGSAC, SIGSOFT and SIGSPATIAL. More than 330 students participated in these competitions.

Students find the SRC highly rewarding. Representative comments include the following:

"Participating in the SRC was an amazing opportunity. It was my first time attending any conference, and it really showed me how to pitch my research project, and interact with other researchers. I will carry this experience with me in my future academic and professional endeavors."

- Michele $\mathrm{Hu}$, Cornell Tech ASSETS 2015

"It was a great opportunity to be able to present at ACM SRC the work developed during a study abroad experience in the USA. Working with students from other places with different backgrounds was an incredible experience. That was my first time presenting at a conference and it felt great to expose our research, discuss it, and get invaluable feedback. It was an amazing chance to train my research pitch and share ideas with others."

- Clarissa Tuxen,

Fluminense Federal University Grace Hopper 2016

To learn more about the SRC, visit http://src.acm.org/.

We acknowledge the dedication of the many volunteers who make the SRC work. Each participating conference has a student research competition chair and program committee who review the papers submitted by the students. This committee and additional volunteers judge the posters and presentations at the conference venue to select the winners. Evelyne Viegas at Microsoft supports the SRC program; she replaces Judith Bishop who was a dedicated Microsoft volunteer for many years. Finally, Nanette Hernandez of ACM handles all logistical aspects of running the SRC, such as sending materials to conference sites around the world, and handles the interactions with students and conference volunteers.

Laurie Williams, a professor of computer science in the College of Engineering at North Carolina State University, Raleigh, and Doug Baldwin, a professor of mathematics at the State University of New York at Geneseo, serve as co-chairs of the ACM Student Research Competition.

Copyright held by authors. 